IOS Press

\title{
Editorial
}

\section{Ignaz Semmelweis}

As the year comes to an end, JPRM enters its tenth year of publication. The United States has elected a new president, England voted to exit the European Union, and the ZIKA virus was discovered in South America. As change abounds worldwide, health care change also seems constant. Regardless of the country, place, time, and/or politics, our collective goal of providing patient care either on an individual or public health basis should not and must not change. Ignaz Semmelweis, known for the Semmelweis Effect - the tendency to reject new evidence that contradicts established norms - was an obstetrician at the Vienna General Hospital in the mid 1800's and provides an example of how systems and individuals all struggle with change.

I first learned of Ignaz Semmelweis in my residency. Some may consider that disappointing as his discovery is practiced routinely every day by hundreds of millions of people. The Semmelweis School of Medicine in Budapest, Hungary was renamed in his honor. He discovered that simply washing hands and instruments between each patient saved lives. This was a radical and antiestablishment position that few accepted at the time. Typically, physicians would perform an autopsy in the morning, wipe their hands on their bloody gowns, and move to the next patient - one after another - never having washed their hands or their instruments between patients or procedures.

As a new physician, Semmelweis noticed that women who delivered children by doctors died at a much higher rate than women who delivered by midwife. Physicians, all of whom were men, trained in one ward and midwives in another. After intensive review, he deduced that the difference was the doctors were performing autopsies and the midwives were not. He speculated that there were cadaveric particles of the autopsy on the doctors' hands and that these were causing what was known as "childbed fever" or "puerperal."

Semmelweis started having his medical team wash everything with chlorine - hands and instruments - between each delivery and after each autopsy. And do- ing so had dramatic results. Many women survived because of his efforts and he was labeled the "savior of mothers", reducing their death rate in the hospital from 18-percent to less than two-percent.

Unfortunately, many of his colleagues dismissed his theory and he was dismissed from the hospital. He moved to Budapest where he practiced for six years, married, and published his findings. He was adamant that he was correct and tried to educate more physicians regarding what he thought and knew was "best practice". His published manuscript "The etiology, concept and prophylaxis of childbed fever" received little recognition. Some thought he had Alzheimer's or a mental health condition and he was committed to an asylum. He apparently was beaten by the guards and died as a young man only two weeks later, ironically maybe by a wound infection.

Louis Pasteur's germ theory later proved that Semmelweis was correct. While he thought cadaveric pieces were causing the deaths, it was actually bacteria. Semmelweis believed he was correct, was ridiculed for the changes he proposed, and was later proven correct by new discoveries. Thus, the Semmelweis Effect.

\section{References}

[1] http://semmelweis.org/about/dr-semmelweis-biography/.

[2] https://www.britannica.com/biography/Ignaz-Semmelweis.

[3] http://fn.bmj.com/mwg-internal/de5fs23hu73ds/progress?id= KGcE9OwCFOyulj1Bgd8y5RYjGC5GXyo-uxp6mwaswsw.

[4] Semmelweis I. The etiology, concept and prophylaxis of childbed fever. Translated by K Codell Carter. Madison: The University of Wisconsin Press, 1983.

\section{Introduction to the open issue}

Please consider this an introduction to our final issue of 2016. We have five original articles, a commentary and a review article.

In the "Current Conservative management and classification of club foot: A review," Balansanar et al. provide a nice summary regarding the historical changes and current treatment in clubfoot management. Prior to 
Ponsetti's conservative management of the club foot, the majority of newborns with club feet were repaired using a surgical technique. Ponsetti had been practicing his casting technique since the 1950's with great success. He had followed and published about his cohort of children, many of whom were now adults, and the success that he had. It took over 40 years for his technique to become widely accepted and is now the preferred conservative management for treatment of clubfeet.

"Traumatic amputations in children and adolescents: A demographic study from a tertiary care center in Northern India" characterizes the demographics of amputations in this area. Road traffic and train accidents accounted for approximately $70 \%$ of all amputations. The authors appropriately emphasize that injury prevention in developing countries like India should be widely publicized to prevent such accidents. These should be continued priorities in all countries worldwide.
The prevalence of plagiocephaly in neonatal brachial plexus palsy is a concern among this population. Tang et al. found that $64 \%$ of their subjects had plagiocephaly. Thus, the importance of screening patients with brachial plexus palsy for plagiocephaly needs further study for incorporation to clinical practice. In "Physical activity and walking performance: Influence on quality of life in ambulatory children with cerebral palsy (CP)", Mann and her colleagues conclude that physical activity, walking performance and level of participation in daily life are associated with various domains of quality of life. This is followed by Ridosh et al.'s article on the quality of life in parents of adolescents and young adults with and without Spina Bifida. They conclude that post depressive symptoms, family stress, and resources should be evaluated routinely, especially for those with low income in addition to other findings.

Sincerely,

Jacob A. Neufeld, MD, MSPH

Editor in Chief, Journal of Pediatric Rehabilitation 